\title{
Surgical strategies for placenta percreta invading the bladder and review of literature
}

\author{
Nikhar Jain * (D, Sujata Patwardhan, Hitesh Jain and Bhushan Patil
}

\begin{abstract}
Background: Major obstetric hemorrhage is the leading cause of maternal morbidity and mortality. In rare cases, lifethreatening hematuria in pregnant women may result from invasion of the bladder by the placenta. We present our experience with 18 cases of placenta percreta with suspected bladder invasion.

Methods: It is a retrospective single-center study conducted over a period of 3 years. Total 18 patients of radiologically diagnosed placenta percreta were included in the study. All patients who are at risk for placenta percreta underwent prenatal sonograms. Patients of Placenta Accreta Spectrum presenting electively also underwent MRI pelvis. Elective patients who were high risk of placenta percreta underwent bilateral placement of the balloon catheter in internal iliac artery. In case of doubt regarding bladder invasion, patient underwent anterior cystotomy and posterior wall of the bladder was examined and proximity of the ureteric orifice to the placenta and amount of involvement of bladder wall was assessed. Ureteric catheter placement was used as adjuncts depending on the proximity of placental invasion with ureteric orifice. Postoperative outcomes in the form of maternal morbidity, maternal mortality, fetal mortality, postoperative bleeding, bladder status, vesicovaginal fistula, bladder capacity were all evaluated.
\end{abstract}

Results: In our series, 17 cases all cases were diagnosed preoperatively by antenatal ultrasound and MRI. Only one patient presented with hematuria. Only in one patient, we attempted separation of placenta from bladder wall, and it resulted in profuse bleeding, and in rest, we excised the involved bladder. Partial cystectomy was done in $33.4 \%$ patients, $27 \%$ patients required bilateral placement of ureteric catheter due to proximity to the ureteric orifice. $33.4 \%$ patient underwent bilateral internal iliac artery ligation or balloon placement. Clot evaluation was needed in one patient. Intraoperatively — 39\% patients had uterus adhered to the bladder but no placental invasion into the bladder. One patient was managed with obstetric hysterectomy and methotrexate followed by clot evacuation and bilateral internal iliac artery ligation at a later date. One (5.6\%) patient developed vesicovaginal fistula in postoperative period. There was one (5.6\%) maternal mortality with no fetal mortality. On follow-up, patient had good bladder capacity, 3 weeks after the surgery.

Conclusion: MRI done preoperatively can help us guide regarding the extent or severity of placental invasion. Intraoperatively, anterior cystostomy should be done in suspected placenta percreta. Grade I or II accrete/percreta patients can be managed conservatively. Partial cystectomy with placement of bilateral ureteric catheter is safer and less morbid approach in tackling placenta percreta invading the bladder with mucosal involvement.

Keywords: Placenta percreta, Bladder invasion by placenta percreta, Obstetric hystrectomy, Placenta accreta

*Correspondence: nikhar.aryan@gmail.com

Seth G. S. Medical College and King Edward Memorial Hospital, 8th Floor MSB, Acharya Dhonde Marg, Mumbai 400012, India

\section{Background}

Normally, a layer of decidua separates the chorionic villi and the myometrium (the inner layer of the uterus) at the site of placental implantation. In placenta percreta, the chorionic villi penetrate the myometrium completely with possible invasion to the adjacent urogenital organs. 
Major obstetric hemorrhage is the leading cause of maternal morbidity and mortality. Placenta percreta is a subgroup of placenta accrete spectrum. Incidence of percreta was 1 in 533 pregnancies for the period of 1982-2002 [1]. The incidence has increased from $0.12 \%$ to $0.31 \%$ in the last 30 years because of rise in Cesarean sections. [2] Out of all cases of Placenta accreta only 5\% are of placenta percreta [3]. The invasion of the bladder is rare with only limited case reports. Patient with placenta percreta usually present with vaginal bleeding and lower abdominal pain. Hematuria as a presentation is rare (25\%) even in percreta with invasion of the bladder [4]. Placenta percreta is a potentially fatal condition and rate is proportional to involvement of surrounding structures.

Techniques for optimal management of placenta percreta invading bladder still remains controversial due to lack prospective randomized control trials on treatment and long-term outcomes. This review aims to consolidate all the available research and highlight the role of multidisciplinary approach and formulate best practice guidelines.

\section{Methods}

Aim of this study was to assess the clinical profile of placenta percreta with bladder invasion and to formulate best clinical practice guidelines in management of bladder invasion by percreta. It is a retrospective single-center study conducted over a period of 3 years. Total 18 patients of radiologically diagnosed placenta percreta with suspected bladder invasion or adhesion were referred to us from the department of gynecology and all were included in the study. All the patients were evaluated with detailed history and examination. Special emphasis was given pertaining to the presence of risk factors for placenta percreta, urological symptoms like hematuria, any past urological disease and prior urological intervention. Detailed evaluation for fetal and maternal wellbeing was done by the gynecologist. All patients who are at risk for placenta percreta underwent prenatal sonograms. Patients were diagnosed with placenta accrete spectrum based on the imaging criteria laid by Baughman et al. which includes placenta previa, lacunae, abnormal color Doppler imaging patterns, loss of the retroplacental clear space, reduced myometrial thickness and irregular bladder wall [5]. Patients presenting electively for antenatal check-up if found to have placenta accrete or percreta also underwent MRI of pelvis to look for possible bladder invasion. MRI features of placenta accrete and percreta were graded as:

1. Lumpy contour, rounded edges, uterine bulging, and placenta previa.
2. Heterogeneous parenchymal signal intensity and the presence of dark, irregular and thick intraplacental bands.

3. Full thickness gap of myometrial signal with loss of fat plane between the placental tissue and adjacent pelvic organs

4. Placental signal disrupting the hypointense line of the bladder.

5. Involvement of the trigone area, bladder neck, ureter involvement.

Urological reference was taken for both planned and emergency surgeries in case of percreta with suspected bladder invasion.

All patients underwent elective supra-vesical classical Cesarean section. Elective patients who were high risk of placenta percreta were evaluated by the interventional radiologist and few patients underwent bilateral placement of the balloon catheter in internal iliac artery. Blood bank physician was informed, cross-matching, and typing of the blood and blood products was done. Adequate reserve of the blood products kept ready.

Balloon placement of bilateral internal iliac artery was done under the discretion of Interventional radiologist and obstetrician based on the severity of bladder invasion, presentation and affordability of the patient.

Obstetric management was done prior to intervening for the bladder. Safe delivery of the fetus was ensured. All obstetric findings were noted. After anterior hysterotomy, placental location was confirmed. Patient was then handed over to urologist for retro-vesical dissection between the posterior bladder wall and anterior uterine wall. Placenta and relation to the bladder was examined. In case no obvious placenta percreta was found, only adhesiolysis was done. In case of only small focal invasion not invading the mucosa of the bladder, often seen after anterior cystotomy placenta separation was attempted. In case of gross invasion of placenta with the bladder, partial cystectomy was done. In case of doubt regarding bladder invasion, patient underwent anterior cystotomy and posterior wall of the bladder was examined. If after anterior cystotomy gross invasion of the bladder by percreta was found; proximity of the ureteric orifice to the placenta and amount of involvement of bladder wall was assessed. Bilateral placement of ureteric catheter was done based on the area of bladder wall involvement and proximity to orifice. Excision of the placenta along with the posterior bladder wall was done using sharp dissection. Hemostasis is achieved and bladder wall closed in two layers using polyglycolic acid sutures. Patients in whom uterus was densely adhered to the bladder an en bloc resection of the posterior bladder wall with uterus was done. Ureteric 
catheter placement was used as adjuncts depending on the proximity of placental invasion with ureteric orifice.

Specimen was sent for histopathological confirmation of placenta percreta invasion into bladder. Postoperative outcomes in the form of maternal morbidity, maternal mortality, fetal mortality, postoperative bleeding, bladder status, vesicovaginal fistula, bladder capacity was all evaluated. At 3 weeks, a cystogram was done in patients who underwent partial cystectomy to assess for leak and bladder capacity.

\section{Result}

Mean maternal age at diagnosis was 29 years and mean fetal age at diagnosis was 28 weeks. Out of these 18 patients, four out of 18 patients presenting directly in the emergency, 3 patient had premature onset of labor, 4 patients had severe vaginal bleeding, 2 among patients had shock as shown in the figure. These symptoms were not mutually exclusive. Routine blood investigation was done along with fetal sonography in these patients. Findings of low-lying placenta with loss of retroplacental space was noted.

Rest 14 out of 18 patients were minimally symptomatic at diagnosis and were followed up regularly in the antenatal period. Among these patients, 3 had minimal vaginal bleeding, 10 patient had lower abdominal pain at presentation. 1 patient was asymptomatic at presentation but developed hematuria during antenatal period. All patients had history of previous Cesarean section and were multiparous. Three patient had prior Uterine curettage, one patient had history of endometriosis, and one underwent manual placental removal in the past. All patients underwent sonographic examination for fetal well-being and noted deficiency of retroplacental sonolucent zone, Vascular lacunae, myometrial thinning and interruption of bladder interface. Findings were corroborated by color doppler. Six out of 18 patients were sonography was equivocal underwent MRI pelvis. MRI findings vis-a-vis non-visualization of the decidua separating placenta from myometrium and invasion into and beyond myometrium was seen.

22.2\% (4/18) patients underwent emergency Cesarean section and rest of the patient had elective Cesarean section. None of the patient had preoperative cystoscopy or ureteric catheter placement. Fetal safety was ensured and fetus was delivered before any urological intervention. All patients underwent supra-vesical Classical cesarean section. Seventeen out of 18 patient underwent obstetric hysterectomy at the time of $\mathrm{C}$ section.

Among the rest of the patients, six out of 18 patients had preoperatively placed balloon catheters in the internal iliac arteries. Total mean blood loss was $3320 \mathrm{ml}$. Blood loss with balloon occlusion of internal iliac artery was $1850 \mathrm{ml}$ which significantly lower than without balloon placement $(4050 \mathrm{ml})$. Mean duration of surgery was $3.12 \mathrm{~h}$ and was significantly different between emergency and elective cases.

\begin{tabular}{|c|c|c|c|}
\hline \multicolumn{4}{|c|}{$\begin{array}{l}\text { Intraoperative observations of radiologically diagnosed placenta } \\
\text { percreta }\end{array}$} \\
\hline Diagnosis & $\begin{array}{l}\text { Percreta with } \\
\text { full thick- } \\
\text { ness bladder } \\
\text { invasion }\end{array}$ & $\begin{array}{l}\text { Percreta with } \\
\text { bladder abut- } \\
\text { ment 4/18-22\% }\end{array}$ & $\begin{array}{l}\text { Placenta } \\
\text { accreta } \\
\text { 7/18-39\% }\end{array}$ \\
\hline Frequency & $39 \%$ & $22 \%$ & $39 \%$ \\
\hline Management & $\begin{array}{l}\text { Placental } \\
\text { separation } \\
\text { and closure } \\
\text { Partial } \\
\text { cystectomy } \\
(33.4 \%)\end{array}$ & $\begin{array}{l}\text { Placental separa- } \\
\text { tion with or } \\
\text { without wall } \\
\text { reinforcement }\end{array}$ & Adhesiolysis \\
\hline
\end{tabular}

Placental separation was attempted but failed in one patient. This patient underwent partial cystectomy and hysterectomy at a later date. Bilateral ureteric catheter was placed in 5/6 undergoing partial cystectomy. The histopathology report correlated with intraoperative findings in all patient. On histopathology in patients with bladder invasion chorionic villi are present in contact with smooth muscle fibers. Rest of the histopathological findings are similar to accrete.

Ureteric catheters were removed after 4-6 days of surgery. Among patients of placenta percreta with bladder invasion, there was no fetal mortality, one maternal mortality at POD 2. One patient developed vesicovaginal fistula. Two patients developed per vaginal bleeding which responded to conservative management. One patient developed hematuria at 11th day postoperatively. Rest of the patient had uneventful course. On followingup patients of partial cystectomy, all had a normal cystogram with no leak and good bladder capacity, 3 weeks after the surgery except one patient who developed vesicovaginal fistula.

One patient had a prolonged hospital course. This patient, 33 years, old multiparous female, presented asymptomatically, was diagnosed as placenta percreta invading the bladder by ultrasonography and MRI during routine antenatal checkup at 30 weeks of gestation. Patient was kept under observation in view high-risk pregnancy and anticipated complications of placenta percreta. Patient developed painless hematuria with clots at 32 weeks of gestation. Patient was managed by bladder irrigation for the same. Patient was managed conservatively till 36 weeks. Preoperative Bilateral internal iliac balloon occluding catheters were placed. Patient was posted for elective Cesarean section. Intraoperatively, placental separation was attempted but failed due to gross invasion of the placenta. Hemostasis was 
achieved and decision taken to conservatively manage the uterus and bladder. On 11th postoperative day, patient developed gross hematuria with bladder clots. Emergency Cystoscopy was done; diffuse ooze was noted from the posterior wall of the bladder at the site of placental invasion. Around $200 \mathrm{cc}$ of clot was evacuated. Inj. Methotrexate could not be given due to fever. Hematuria recurred after 6 days. Repeat cystoscopy was done and clot evacuation attempted cystoscopically but failed. Open-clot evacuation done in view of organized bladder clots. Inj. Methotrexate was given $50 \mathrm{mg}$ alternate day $(\times 3$ doses). Urine was clear postoperatively, 2 units PCV transfused. Beta-hCG levels were monitored. Five days later, she developed third episode of hematuria. Repeat MRI was done at this time. MRI was suggestive of clots adhered to the placenta percreta in the bladder. Patient underwent exploratory laparotomy with bladder clot evacuation. Decision was taken to perform partial cystectomy with hysterectomy due to repeated episodes of hematuria, falling hematocrit not responding to conservative management and avoiding complications of massive blood transfusion with bilateral internal artery ligation. DJ stents were placed. She required six blood transfusions, four bags of fresh frozen plasma, and one bag of platelets. Two units of PCV were transfused postoperatively. DJ stent removed after 4 weeks.

One patient was referred 2 months postdelivery in view of hematuria. She was a 35-year-old primigravida found to have placenta totally invading uterine myometrium. Placenta was left in situ as it was adhered and was managed with methotrexate. She had recurrent hematuria at 2 months postpartum, initially managed conservatively with clot evacuation. MRI Pelvis was suggestive of placental mass approximately of size $19 \times 10 \times 13 \mathrm{~cm}$ extending into superior wall of urinary bladder and encasing left distal ureter with upstream dilatation. Patient underwent cystoscopy with Left double J stenting. Patient treated as Placenta percreta and received three doses of Methotrexate $50 \mathrm{mg}$ every alternate day. Double J stent was removed after 3 months. Her betaHuman Chorionic Gonadotropin levels dropped down to $2 \mathrm{mIU} / \mathrm{ml}$.

\section{Discussion}

There is a rise in incidence of placenta percreta over the last 3 decades due to rise in number of cesarean sections [6]. Even in cases of bladder invasion by placenta percreta, gross hematuria is rare, and it occurred in only 6 of the 27 previously reported cases from 1965 to 2000 [7]. The most common presentation of percreta with bladder invasion is still lower abdomen pain and per vaginal bleeding. Other rare differentials for painless hematuria in pregnancy include nutcracker phenomenon, Youssef syndrome, paragangliomas, renal arterio-venous malformations, molar pregnancy, etc. Cystoscopy may be required to identify the exact cause of hematuria. Cystoscopy may often show posterior bladder wall abnormalities but we should avoid taking biopsy from these lesions [4]. Limitations of cystoscopy in this setting include-difficulty due to gravid uterus, biopsy of this lesions cannot be done limiting the diagnostic power of cystoscopy and many patients present as emergency.

Important risk factors for placenta percreta include previous Cesarean sections, advanced maternal age, multiparity, uterine curettage/surgery, and Asherman syndrome. All patients were multiparous and had history of Cesarean section in comparison to $80 \%$ in study by Tantbiroin et al. [8].

Diagnosis is usually by antenatal USG and/or MRI. Overall good accuracy with a sensitivity of $81.2 \%$ and a specificity of $98.9 \%$ [9]. Findings in ultrasound include: placental lacunae within the placental substance, irregular border between the bladder and myometrium, thin myometrium, and loss of retroplacental hypoechoic myometrial zone, thinning or disruption of the linear hyperechoic boundary echo representing the uterine serosa, and its interface with the posterior bladder. Doppler ultrasonography will often reveal turbulent blood flow extending from the placenta to surrounding tissues. MRI has a definite role when the ultrasound evaluation is equivocal or for patients with high clinical risk factors for placenta accreta. The most reliable MR findings are uterine bulging, heterogeneous placenta, and placental bands. Focal interruptions in the hypointense myometrial border may also be helpful.

Multidisciplinary management at a tertiary care in this high-risk pregnancy should be sought with specific role defined for each specialty. Obstetricians are usually the first point of contact with the patient. Proper counselling, antenatal checkups, fetal scans are performed. They also decide the optimum timing of delivery. Radiologist helps in diagnosis, fetal scans, and VIR guided placement of balloon occlusion catheters in internal iliac artery. Blood bank physician assists by stocking and providing adequate blood products during surgery. Urologist have a role in management of hematuria preoperatively. Intraoperatively obstetrician performs Cesarean section, urologist takes care of the bladder, and radiologist helps in intraoperative embolization of internal iliac artery. Anesthetist and neonatologist also form important members of the team.

Delivery should not to exceed 34-35 weeks of gestation because it significantly increases risk of severe hemorrhage [10]. Most patients can be planned for elective Cesarean section during this time. Severe antepartum hemorrhage not responding to conservative measures 
require emergent intervention. The rate of emergency Cesarean section is comparable to the studies conducted by Bretelle et al. [11]. O'Brien et al. reported that almost all cases of placenta percreta with bladder involvement had undergone either total or subtotal hysterectomy [12].

Preoperative bilateral occlusion of internal iliac artery significantly decreases blood loss. Dubois et al. recommended prophylactic bilateral internal iliac ligation or preoperative cannulation of the hypogastric arteries with an occlusion balloon, and intraoperative embolization to decrease blood loss during hysterectomy [13]. Jaraquemada et al. performed a uterine conservation by early vascular control at the level of infrarenal aorta followed by uterine and vesical repair [14]. Blood loss with bilateral internal artery management is around $45 \%$ less in our study comparable to study by Clausen et al. [15].

Urologists are usually consulted after a life-threatening emergency situation has already arisen. Massive hemorrhage occurs when attempts are made to separate the adherent placenta from the bladder. Price et al. suggested a modified posterior approach with en bloc removal of the posterior bladder wall when it is impossible to separate the bladder from the lower uterine segment. Abbas et al. recommend performing [16] anterior bladder wall cystotomy to define dissection planes and determining whether posterior bladder wall resection is required [7]. In case of no mucosal involvement and minimal serosal involvement or grade I/II according to MRI, placental separation can be attempted.

Our observation mainly concerns with management of extensive bladder involvement. In case of extensive mucosal involvement by placenta or Grade II/IV/V according to MRI, then placental separation should not be attempted. Anterior cystostomy should be performed in all such cases to evaluate the extent of bladder involvement.

Our study is a retrospective and a non-randomized study of this complex condition. Randomization is usually not possible or difficult to perform in view of emergent nature of this condition.

\section{Conclusion}

MRI done preoperatively can help us guide regarding the extent or severity of placental invasion based on the above-mentioned features. We recommend (a) preoperative occlusion of the internal iliac artery should be done in all the cases as it reduces intraoperative blood loss by around $45 \%$. (b) Intraoperatively, anterior cystostomy should be done to evaluate for mucosal invasion of percreta to avoid extreme blood loss by not attempting placental separation. Partial cystectomy with placement of bilateral ureteric catheter is safer and less morbid approach. None of our patient had anterior wall or trigonal involvement and therefore, no comments could be made on total cystectomy or diversion. Few patients might present postdelivery and should be managed according to the presentation and site of involvement.

\section{Abbreviations}

HCG: human chorionic gonadotropin; IIA: internal iliac artery; Inj.: injection; ml: milliliter; MRI: magnetic resonance imaging; PCV: packed cell volume; U: units.

\section{Acknowledgements}

Department of Gynaecology, KEM Hospital.

\section{Authors' contributions}

NJ performed conception, drafted the work, and revision of work. SP designed the work, interpreted the data. HJ performed acquisition and analysis and revision of work. BP interpreted the data. All authors have read and approved the manuscript being submitted for review.

\section{Funding}

The study was not supported financially by anybody.

Availability of data and materials

All data are kept in Medical Records department of KEM hospital.

\section{Ethics approval and consent to participate}

Ethics committee name-Institutional Ethics Committee, KEM hospital. It is retrospective observational study based on the standard clinical practices with no deviation from it and hence the ethics approval is waived off by the institutional Ethics committee.

\section{Consent for publication}

Written informed consent taken from all the patients.

\section{Consent to participate}

Informed written consent was provided by all participants to participate in this study.

\section{Consent for publication}

Informed written consent was provided by all participants to participate to publish them in the study.

\section{Competing interests}

The authors declare that they have no competing interests.

Received: 15 November 2019 Accepted: 11 September 2020 Published online: 06 November 2020

References

1. Wu S, Kocherginsky M, Hibbard JU (2005) Abnormal placentation: twenty-year analysis. Am J Obstet Gynecol 192:1458-1461

2. Zhang D, Yang S, Hou Y, Su Y, Shi H, Gu W (2017) Risk factors, outcome and management survey of placenta accreta in 153 cases: a five-year experience from a hospital of Shanghai, China. Int J Clin Exp Med 10(8):12509-12516

3. Konijeti R, Rajfer J, Askari A (2009) Placenta percreta and the urologist. Rev Urol 11(3):173

4. Takai N, Eto M, Sato F et al (2005) Placenta percreta invading the urinary bladder. Arch Gynecol Obstet 271:274-275

5. Baughman WC, Corteville JE, Shah RR (2008) Placenta accreta: spectrum of US and MR imaging findings. Radiographics 28(7):1905-1916

6. Hudon L, Belfort MA, Broome DR (1998) Diagnosis and management of placenta percreta: a review. Obstet Gynecol Surv 53:509-517

7. Abbas F, Talati J, Wasti S et al (2000) Placenta percreta with bladder invasion as a cause of life threatening hemorrhage. J Urol 164:1270-1274 
8. Tantbirojn P, Crum CP, Parast MM (2008) Pathophysiology of placenta accreta: the role of decidua and extravillous trophoblast. Placenta 29:639-645

9. Pagani G, Cali G et al (2018) Diagnostic accuracy of ultrasound in detecting the severity of abnormally invasive placentation: a systematic review and meta-analysis. Acta Obstet Gynecol Scand 97(1):25-37

10. Robinson BK, Grobman WA (2010) Effectiveness of timing strategies for delivery of individuals with placenta previa and accreta. Obstet Gynecol 116:835-842

11. Bretelle F, Courbière B, Mazouni C, Agostini A, Cravello L, Boubli L, Gamerre M, D'Ercole C (2007) Management of placenta accreta: morbidity and outcome. Eur J Obstet Gynecol Reproduct Biol 133(1):34-39

12. O'Brien JM, Barton JR, Donaldson ES (1996) The management of placenta percreta: conservative and operative strategies. Am J Obstet Gynecol 175:1632-1638

13. Dubois J, Garel L, Grignon A, Lemay M, Leduc L (1997) Placenta percreta: balloon occlusion and embolization of the internal iliac arteries to reduce intraoperative blood losses. Am J Obstet Gynecol 176:723-726
14. Palacios Jaraquemada JM, Pan G (2000) Uterine conservation in patient with consecutive double placenta percreta. Acta Obstet Gynecol Scand 79:900-901

15. Clausen C, Lönn L, Langhoff-Roos J (2014) Management of placenta percreta: a review of published cases. Acta Obstet Gynecol Scand 93(2):138-143

16. Price FV, Resnik E, Heller KA et al (1991) Placenta previa percreta involving the urinary bladder: a report oftwo cases and review of the literature. Obstet Gynecol 78:508

\section{Publisher's Note}

Springer Nature remains neutral with regard to jurisdictional claims in published maps and institutional affiliations.

\section{Submit your manuscript to a SpringerOpen ${ }^{\circ}$ journal and benefit from:}

- Convenient online submission

- Rigorous peer review

- Open access: articles freely available online

- High visibility within the field

- Retaining the copyright to your article

Submit your next manuscript at $\boldsymbol{\nabla}$ springeropen.com 